\title{
Modelling of a Small Unmanned Aerial Vehicle
}

\author{
Ahmed EA ${ }^{1 *}$, Hafez $\mathrm{A}^{1}$, Ouda $\mathrm{AN}^{2}$, Ahmed $\mathrm{HEH}^{3}$ and Abd-Elkader $\mathrm{HM}^{1}$ \\ ${ }^{1}$ Electrical Engineering Department, Shoubra Faculty of Engineering, Benha University, Egypt \\ ${ }^{2}$ Military technical College, Cairo, Egypt \\ ${ }^{3}$ Communication Engineering Department, Faculty of Electronic Engineering, Menoufia University, Menouf, Egypt
}

\begin{abstract}
Unmanned aircraft systems (UAS) are playing increasingly prominent roles in defense programs and defense strategies around the world. Technology advancements have enabled the development of it to do many excellent jobs as reconnaissance, surveillance, battle fighters, and communications relays. Simulating a small unmanned aerial vehicle (SUAV) dynamics and analyzing its behavior at the preflight stage is too important and more efficient. The first step in the UAV design is the mathematical modeling of the nonlinear equations of motion. A survey with a standard method to obtain the full non-linear equations of motion is utilized, and then the linearization of the equations according to a steady state flight condition (trimming) is derived. This modeling technique is applied to an Ultrastick-25e fixed wing UAV to obtain the valued linear longitudinal and lateral models. At the end, the behavior of the states of the non-linear UAV and the resulted linear model is compared by applying a doublet signals in the control surfaces to check the matching between them.
\end{abstract}

Keywords: Equations of motion; Linearization; Modeling; Nonlinear model; UAV

\section{Nomenclature}

$\begin{array}{ll}\left(\mathrm{x}^{\mathrm{i}}, \mathrm{y}^{\mathrm{i}}, \mathrm{z}^{\mathrm{i}}\right) & \begin{array}{l}\text { inertial frame axes } \\ \left(\mathrm{x}^{\mathrm{v}}, \mathrm{y}^{\mathrm{v}}, \mathrm{z}^{\mathrm{v}}\right)\end{array} \\ \left(\mathrm{x}^{\mathrm{b}}, \mathrm{y}^{\mathrm{b}}, \mathrm{z}^{\mathrm{b}}\right) & \text { vehicle frame axes } \\ \phi, \theta, \psi & \text { body frame axes } \\ \alpha & \text { attitude angles, rad } \\ \beta & \text { Angle of attack } \\ \chi & \text { Side slip angle } \\ \chi_{c} & \text { Course angle } \\ & \text { Crab angle }\end{array}$

$\gamma \quad$ Inertial-referenced flight path angle

$u, v, w \quad$ Inertial velocity components of the airframe projected onto $\mathrm{x}^{b}$-axis

$\mathrm{V}_{a} \quad$ Airspeed vector

$\mathrm{V}_{g} \quad$ Ground speed vector

$\mathrm{V}_{w} \quad$ Wind speed vector

$\mathrm{C}_{\mathrm{L}} \quad$ Aerodynamic lift coefficient

$\mathrm{C}_{\mathrm{D}} \quad$ Aerodynamic drag coefficient.

$C_{m_{*}} \quad$ Aerodynamic pitching moment coefficient

$C_{p_{*}} \quad$ Aerodynamic moment coefficient along the $x^{b}$-axis

$C_{q_{*}} \quad$ Aerodynamic moment coefficient along $y^{b}$-axis

$C_{\text {prop }} \quad$ Aerodynamic coefficient for the propeller.

$C_{q_{*}} \quad$ Aerodynamic moment coefficient along the $z^{b}$

$C_{X *} \quad$ Aerodynamic force coefficient along $x^{b}$

$C_{Y_{*}} \quad$ Aerodynamic force coefficient along $y^{b}$

$C_{Z *} \quad$ Aerodynamic force coefficient along $\mathrm{z}^{\mathrm{b}}$

$\delta_{a} \quad$ aileron deflection $\delta_{e} \quad$ elevator deflection

$\delta_{r} \quad$ rudder deflection

$\delta_{t} \quad$ throttle deflection

$f_{\mathrm{D}} \quad$ Force due to aerodynamic drag

$f_{\mathrm{L}} \quad$ Force due to aerodynamic lift

$m_{\mathrm{b}} \quad$ External moment applied to the airframe

$l, m$, and $n$ the components of $m_{\mathrm{b}}$ in $\mathrm{m}^{\mathrm{b}}$

g Gravitational acceleration $\left(9.81 \mathrm{~m} / \mathrm{s}^{2}\right)$

A. Products of the inertia matrix

h Altitude

$\rho \quad$ Density of air

J The inertia matrix

$\mathrm{J}_{\mathrm{x}}, \mathrm{J}_{\mathrm{y}}, \mathrm{J}_{z}$, and $\mathrm{J}_{\mathrm{xz}} \quad$ Elements of the inertia matrix

$\mathrm{k}_{\text {motor }} \quad$ Constant that specifies the efficiency of the motor

$S_{\text {prop }} \quad$ Area of the propeller

UAS Unmanned aircraft systems

SUAV Simulating a small unmanned aerial vehicle

AOA Angle of attack

*Corresponding author: Ahmed EA, Shoubra Faculty of Engineering, Electrical Engineering Department, Benha University, Egypt, Tel: 201007124097; E-mail: eng_medoelbanna@yahoo.com

Received December 25, 2014; Accepted January 21, 2015; Published January 28,2015

Citation: Ahmed EA, Hafez A, Ouda AN, Ahmed HEH, Abd-Elkader HM (2015) Modelling of a Small Unmanned Aerial Vehicle. Adv Robot Autom 4: 126. doi: 10.4172/2168-9695.1000126

Copyright: (c) 2015 Ahmed EA, et al. This is an open-access article distributed under the terms of the Creative Commons Attribution License, which permits unrestricted use, distribution, and reproduction in any medium, provided the original author and source are credited. 


\section{Introduction}

Dynamic modeling is an important step in the development and the control of a system as UAV. This model allows the designers to analyze the system; its possibilities and its behavior under various conditions. Especially it's so important for aerial robots where the risk of damage is very high. Thus, the possibility to simulate and tune a controller before implementing it is highly appreciable. A valid linear mathematical model for a fixed wing SUAV is introduced by applying the basic steps of modeling. This paper is organized beginning with introduction section and the other sections are arranged as follows.

$2^{\text {nd }}$ section introduces the coordinate frames which are used to transfer any rigid body from frame to another, the Euler angles $(\psi, \theta, \phi)$ Direct Cosine Matrix (DCM) $[1,2]$. The definition of stability and wind frames. The extracted angles from rotation of object from body frame to the wind frame (angle of attack (AOA), and Sideslip angles $(\alpha, \beta)$ (respectively) [3].

The $3^{\text {rd }}$ section states the basic used parameters of any fixed wing UAV which are used in the aircraft modeling (geometric parameters, inertia, aerodynamic parameters, and control surfaces) [4,5]; these parameters which are obtained from NASA laboratories will be applied to an Ultrastick-25e (Thor) UAV to get the linear mathematical model of this SUAV $[6,7]$.

The $4^{\text {th }}$ section introduces a standard algorithm for an aircraft modeling and converting it from a black box into airframe. It has 12 -state nonlinear equations called equations of motion to describe the motion of the UAV. Beginning from the kinematics, and then adding the force and moment terms expressed in the body frame. The forces applied to the aircraft are the gravity forces, aerodynamic forces, propeller forces. These forces are represented in the body frame, and then converted to the stability frame to get the Lift, Drag, and side forces. Then considering the moments and momentum applied to the aircraft. At last catching the nonlinear equations of motion is the end of this section $[8,9]$.

At $5^{\text {th }}$ section, linearization of the state equations about an equilibrium point (trimming) is applied. At First, we calculate the trimmed values according to the steady state flight condition [2], then decomposition of the dynamic equations into two separated groups (longitudinal and lateral dynamics). The linearization technique for every group is applied separately to derive a linear state space model for longitudinal motion $\left(A_{l o n}, B_{l o n}, C_{l o n}, D_{l o n}\right)$, and a linear state space model for lateral motion $\left(A_{l a p}, B_{l a r}, C_{l a p} D_{l a t}\right)$ according to straight and leveling flight trim conditions [8]. The linearization of the roll dynamics by analytical technique is utilized. At the last of this section a comparison between two techniques is utilized.

At the last section, another comparison between behavior of nonlinear and linear models is done by applying a doublet response to check the matching between them.

\section{UAV Coordination Frames}

In aerospace applications expressing a given vector in terms of a new Cartesian coordinate frame is commonly needed. In this section the descriptions of the various coordinate frames are illustrated starting with Inertial frame. The angles relating the transfer from vehicle frame to the body frame are the yaw $(\psi)$, pitch $(\theta)$ and roll $(\varphi)(\varphi)$. These Euler angles describe the attitude of the aircraft. The angles relating the rotation between the body to the stability frame vice versa, and stability frame to the wind frame are called angle of attack ( $\alpha$ ), and sideslip angle $(\beta)$ respectively. These coordinates are discussed briefly as follows $[1,2,10]$.

\section{Coordinate frames}

Inertial Frame (fi).

This frame is the earth fixed frame and called NED frame.

Vehicle Frame $\left(f^{v}\right)$.

The axes of the vehicle frame as in Figure 1a.

Vehicle-1 Frame $\left(\mathrm{f}^{\mathrm{v}}\right)$.

The rotation of the vehicle- 1 frame extract the heading angle $(\psi)$ right handed is positive as in Figure $1 \mathrm{~b}$.

Vehicle-2 Frame $\left(\mathrm{f}^{\mathrm{v}}\right)$.

The rotation of the vehicle- 2 frame extract the pitching angle $(\theta)$ right handed is positive as in Figure 1c.

- $\quad$ Body Frame $\left(\mathrm{f}^{\mathrm{b}}\right)$.

The rotation of the body frame extract the rolling angle $(\phi)$ or called as bank angle, right handed is positive as in Figure 1d. The transformation matrix from the vehicle frame to the body frame is DCM which is a function of the Euler angles $(\psi, \theta, \phi)$ is.

$$
\begin{aligned}
& R_{v}^{b}(\phi, \theta, \psi)=R_{v 2}^{b}(\phi) \cdot R_{v 1}^{v 2}(\theta) \cdot R_{v}^{v 1}(\psi) \\
& =\left(\begin{array}{lcr}
c \theta c \psi & c \theta s \psi & -s \theta \\
s \phi s \theta c \psi-c \phi s \psi & s \phi s \theta s \psi+c \phi c \psi & s \phi c \theta \\
c \phi s \theta c \psi+s \phi s \psi & c \phi s \theta s \psi-s \phi c \psi & c \phi c \theta
\end{array}\right)
\end{aligned}
$$

Where $\mathrm{c}=\cos , \mathrm{s}=\sin$

The rotation sequence $\psi-\theta-\phi$ is commonly used for aircraft and is just one of several Euler angle systems in use [11]. Euler angles representation suffer from a singularity $(\theta= \pm \pi / 2)$ also known as the "gimbal lock". In practice, this limitation does not affect the SUAV in normal flight mode [12].

\section{- $\quad$ Stability Frame (fs).}

The rotation of stability frame extracts AOA ( $\alpha$ ) which is the lefthanded rotation about the body $\mathbf{y}^{\mathbf{b}}$-axis. The airspeed velocity $(\mathrm{Va})$ is the velocity of the aircraft relative to the surrounding air.

\section{Wind Frame $\left(\mathbf{f}^{\mathrm{w}}\right)$.}

The angle between the velocity vector and the $\mathbf{x}^{\mathbf{b}}-\mathbf{z}^{\mathbf{b}}$ plane is called the side-slip angle and is denoted by $\beta$. Figure 2 illustrates the angles extracted from rotation from the body frame to the stability frame to the wind frame $(\alpha, \beta)$ respectively. The total transformation from the body frame to the wind frame is given by.

$$
\begin{aligned}
& R_{b}^{w}(\alpha, \beta)=R_{b}^{s}(\alpha) \cdot R_{s}^{w}(\beta) \\
& =\left(\begin{array}{ccc}
c \beta c \alpha & -s \beta c \alpha & -s \alpha \\
s \beta & c \beta & 0 \\
c \beta s \alpha & -s \beta s \alpha & c \alpha
\end{array}\right)
\end{aligned}
$$

\section{Wind triangle}

The significant effect of the wind is very important in SUAV. The wind triangle briefly illustrates some relations and definitions can be 

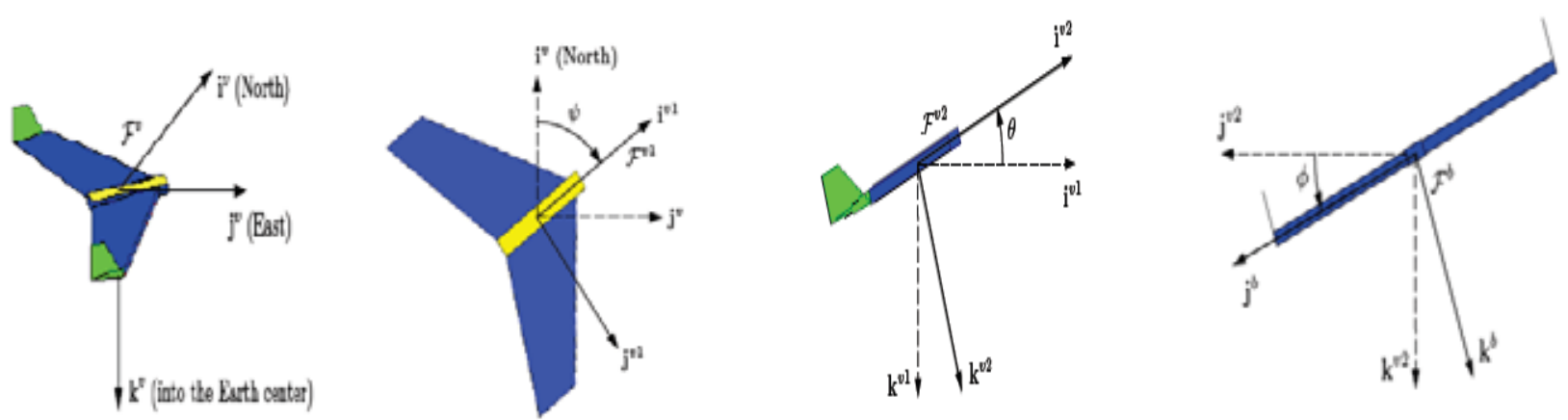

Figure 1: a, b, c, and d respectively: The Euler angles and rotational frames illustrations.

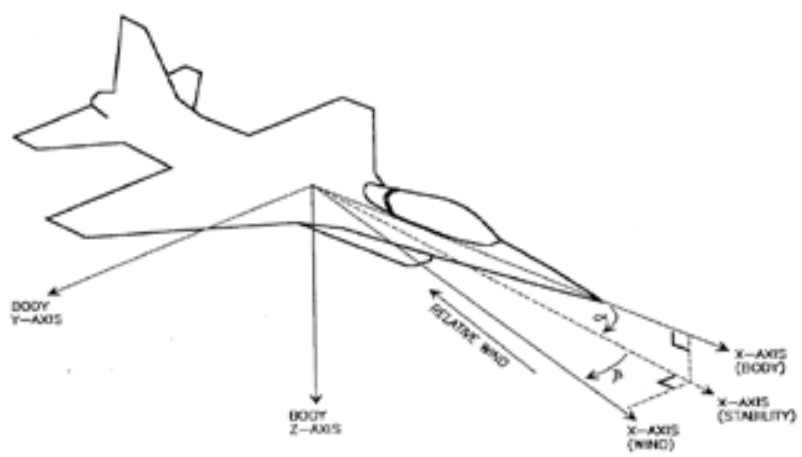

Figure 2: The rotation angles between the body axis and the wind axis.

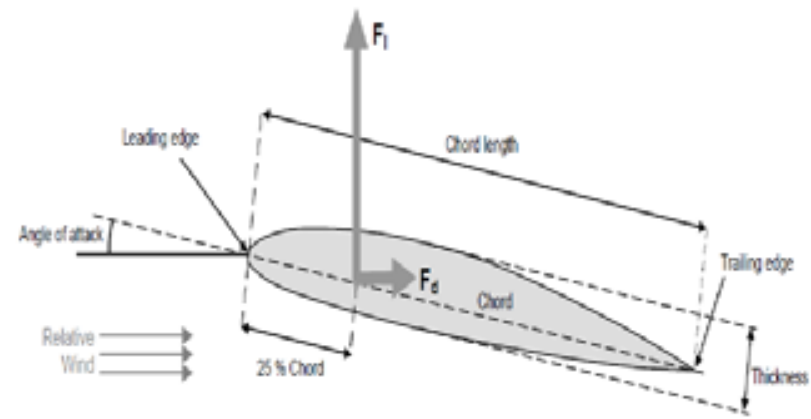

Figure 3: Section of airfoil and the applied lift and drag forces.

considered in the navigation of the SUAV.

The angle between the inertial North $\left(\mathrm{x}^{\mathrm{i}}\right)$ and the inertial velocity vector projected on the horizontal plane is called the course angle $(\mathcal{X})$. The crab angle $\left(\boldsymbol{X}_{\boldsymbol{c}}\right)$ is defined as the difference between the course angle and the heading.

If the wind triangle is projected in the vertical plane another angles can be defined, the flight path angle $(\gamma)$ is the angle between the horizontal plane and the ground velocity $\left(\mathbf{V}_{\mathrm{g}}\right)$, so there are two main angles to transform from body frame to flight path frame $(\chi, \gamma)[6]$.

Notes: In the absence of wind,

- The crab angle $\left(\mathrm{x}_{\mathrm{c}}\right)$ equal zero.

\begin{tabular}{|l|c|c|}
\hline Property & Symbol & The value \\
\hline Wing span & $\mathrm{b}$ & $1.27 \mathrm{~m}$ \\
\hline Wing surface area & $\mathrm{S}$ & $0.3097 \mathrm{~m}^{2}$ \\
\hline Maincord & $\mathrm{C}$ & $0.25 \mathrm{~m}$ \\
\hline Mass & $\mathrm{m}$ & $1.959 \mathrm{~kg}$ \\
\hline Inertia & $\mathrm{J}_{\mathrm{x}}$ & $0.07151 \mathrm{~kg} \cdot \mathrm{m}^{2}$ \\
\cline { 2 - 3 } & $\mathrm{J}_{\mathrm{y}}$ & $0.08636 \mathrm{~kg} \cdot \mathrm{m}^{2}$ \\
\cline { 2 - 3 } & $\mathrm{J}_{z}$ & $0.15364 \mathrm{~kg} \cdot \mathrm{m}^{2}$ \\
\cline { 2 - 3 } & $\mathrm{J}_{x z}$ & $0.014 \mathrm{~kg} \cdot \mathrm{m}^{2}$ \\
\hline
\end{tabular}

Table 1: Some Physical Properties of Ultrastick-25e.

- $\quad$ The sideslip angle $(\beta)$ equal zero.

- $\quad \mathrm{Va}=\mathrm{Vg}$

\section{Fixed Wing UAV Parameters}

This section presents the basic used parameters of the UltraStick25e (Thor). It has a conventional fixed-wing airframe with flap, aileron, rudder, and elevator control surfaces. The maximum deflection of servo actuators equals $25 \mathrm{deg}$ in each direction. The physical details of the aircraft can be found in Table 1, for more details of the University of Minnesota Ultra Stick 25e platform [6,7].

\section{Geometric parameters}

The shape of the airfoil determines its aerodynamic properties, and some of its geometrical parameters. Some of aerodynamic parameters are shown in the Figure 3.

\section{Basic aerodynamic parameters}

The dynamics of the UAV is decomposed into longitudinal and lateral dynamics; each of them has some aerodynamic nondimensional coefficients affect the stability of the aircraft. These coefficients are parameters in the aerodynamic forces and moments equations, and influenced by the airfoil design. A detailed discussion of these coefficients with respect to longitudinal and lateral dynamics existed in $[4,5,8]$.

Longitudinal aerodynamic coefficients: The longitudinal motion acts in the $\mathrm{x}^{\mathrm{b}}-\mathrm{z}^{\mathrm{b}}$ plane which is called pitch plane and affected by the lift force $\left(f_{L}\right)$, Drag force $\left(f_{D}\right)$, and pitch moment $(m)$. The effectiveness of these forces and moments are measured by lift coefficient $\left(C_{L}\right)$, drag coefficient $\left(C_{D}\right)$, and pitch moment coefficient $\left(C_{m}\right)$. These coefficients influenced by the angle of attack $(\alpha)$, pitch angular rate $(q)$, and elevator deflection $\left(\delta_{e}\right)$, but they are nonlinear in the angle of attack; For small 


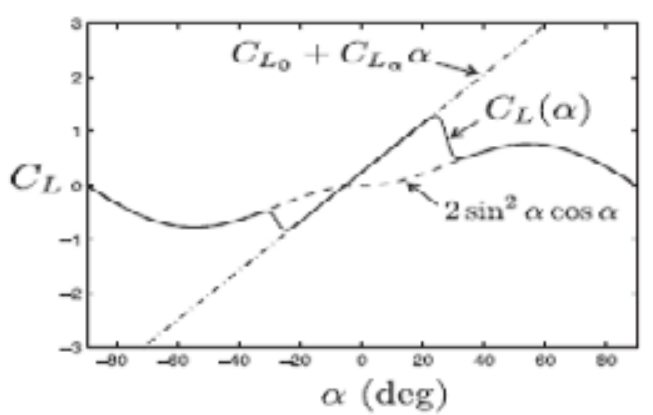

Figure 4: The lift coefficient as a function of $a$ can be approximated by blending a linear function of $\alpha$ (dot-dashed).

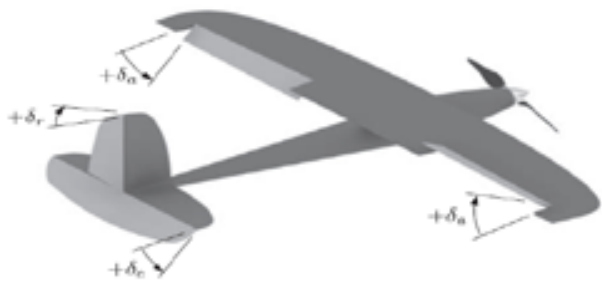

Figure 5: SUAV control surfaces.

$\alpha$ the flow over the wings remain laminar, so no stall conditions will be happened, then we will linearize the equations about this linear zone as in Figure 4.

Lateral aerodynamic coefficients: The lateral motion which is responsible of the yaw and roll motions. It's affected by the side force $\left(f_{Y}\right)$, yaw moment $(\mathrm{n})$, and roll moment (l). The effectiveness of these forces and moments are measured by side force coefficient $\left(C_{Y}\right)$, yaw moment coefficient $\left(C_{n}\right)$, and roll moment coefficient $\left(C_{n}\right)$. These coefficients influenced by sideslip angle $(\beta)$, yaw angular rate $(r)$, roll angular rate $(p)$, aileron deflection $\left(\delta_{a}\right)$, and rudder deflection $\left(\delta_{r}\right)$, but they are nonlinear in these parameters.

All of these coefficients should be determined by wind tunnel. Linear approximations for these coefficients and their derivatives are acceptable for modeling purposes and accurate, the linearization is produced by the first-Taylor approximation, and non-dimensionalize of the aerodynamic coefficients of the angular rates [5].

Note: The effect of Reynolds number and Mach number can be neglected because they are approximately constant in the SUAV dynamics [8].

\section{Fixed wing UAV control surfaces}

As said earlier the designed UAV in this paper is a standard fixed wing UAV with a standard control surfaces; the elevator, the rudder, the aileron, and the thrust with deflections named $\delta_{e}, \delta_{r}, \delta_{a}, \delta_{t}$ respectively, the input controls are shown in Figure 5.

\section{UAV Flight Dynamics}

The first step of design a controlled UAV is to derive the dynamic model. This section will focus on a standard method for deriving the full nonlinear equations of motion of a fixed wing aircraft.

From beginning, several major assumptions are considered.
First, the aircraft is rigid. Although aircraft are truly elastic in nature, modeling the flexibility of the UAV will not contribute significantly to the research at hand. Second, the earth is an inertial reference frame. Third, aircraft mass properties are constant throughout the simulation. Finally, the aircraft has a plane of symmetry. The first and third assumptions allow for the treatment of the aircraft as a point mass [13]

\section{Forces and moments applied on the aircraft}

UAV is subjected to external forces and moments due to gravity, propulsion, and aerodynamics, after applying the newton's second law for translational motion, the applied forces are combined and expressed in the body frame.

$$
f_{b}=\left(f_{x}, f_{y}, f_{z}\right)^{T} \text {. }
$$

For rotational motion the applied moments are combined and expressed in the body frame. For moments,

$$
m_{b}=(l, m, n)^{T} \text {. }
$$

Momentum is defined as the product of inertia matrix $j$ and the angular velocity vector. Due to symmetry of the aircraft about the plane $\mathrm{x}^{\mathrm{b}}-\mathrm{z}^{\mathrm{b}}$, the only inertia used in the modeling is $\mathbf{j}_{\mathbf{x}}, \mathbf{j}_{\mathbf{y}}, \mathbf{j}_{\mathbf{z}}$, and $\mathbf{j}_{\mathrm{x} z}$

$$
\mathrm{j}_{\mathrm{xy}}=\mathrm{j}_{\mathrm{yz}}=0 \text {. }
$$

Six degrees of freedom 12-state equations of motion are obtained, but they are not complete, the external forces and moments are not defined yet. The modeling of the forces and moments can be utilized to get finally the nonlinear 12- state equations of motion.

The gravity $\left(f_{g}\right)$, aerodynamic $\left(f_{a}\right)$, and propeller $\left(f_{p}\right)$ forces are the composition of the total forces applied on the body frame $\left(f_{x}, f_{y}, f_{z}\right)$. Aerodynamic $\left(\mathrm{m}_{\mathrm{a}}\right)$, and propeller $\left(\mathrm{m}_{\mathrm{p}}\right)$ moments is the composition of the total moments applied on the body frame $(\mathrm{l}, \mathrm{m}, \mathrm{n})$ as shown in Figure 6, there are no moments produced by the gravity. The above forces will be represented in the stability frame to get $F_{L}$, and $F_{D}[14]$.

\section{Atmospheric disturbance}

At the existence of wind the atmospheric disturbances with its two components (steady ambient wind, and wind gusts) can be modeled; the steady ambient wind is modeled as a constant wind field, the wind gusts is modeled as a turbulence which is generated by passing white noise linear time invariant filter, the Dryden gust model approximations can be considered in the modeling MIL-F-8785C can be used [9].

\section{Full nonlinear equations of motion}

Finally the following equations of motion are as follows [8].

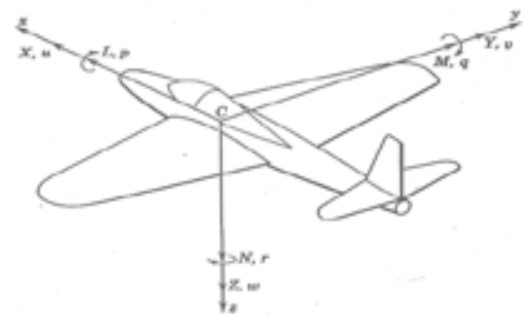

Figure 6: Definitions of UAV body velocities, forces, moments, and angular rates. 


$$
\begin{aligned}
& \dot{p}_{n}=(\cos \theta \cos \psi) u+(\sin \phi \sin \theta \cos \psi-\cos \phi \sin \psi) v+(\cos \phi \sin \theta \cos \psi+\sin \phi \sin \psi) w \\
& \dot{p}_{e}=(\cos \theta \sin \psi) u+(\sin \phi \sin \theta \sin \psi+\cos \phi \cos \psi) v+(\cos \phi \sin \theta \sin \psi-\sin \phi \cos \psi) w \\
& h=u \sin \theta-v \sin \phi \cos \theta-w \cos \phi \cos \theta \\
& \dot{u}=r v-q w-g \sin \theta+\frac{\rho V_{a}^{2} S}{2 m}\left[C_{X}(\alpha)+C_{X_{q}}(\alpha) \frac{c q}{2 V_{a}}+C_{X_{\delta_{e}}}(\alpha) \delta_{e}+\frac{\rho S_{\text {prop }} C_{\text {prop }}}{2 m}\left[\left(k_{\text {moto }} \delta_{t}\right)^{2}-V_{a}^{2}\right]\right] \\
& \dot{v}=p w-r u+g \cos \theta \sin \phi+\frac{\rho V_{a}^{2} S}{2 m}\left[C_{Y_{0}}+C_{Y_{\beta}} \beta+C_{Y_{p}} \frac{b p}{2 V_{a}}+C_{Y_{r}} \frac{b r}{2 V_{a}}+C_{Y_{S a}} \delta_{a}+C_{Y_{s i}} \delta_{r}\right] \\
& \dot{w}=q u-p v+g \cos \theta \cos \phi+\frac{\rho V_{a}^{2} S}{2 m}\left[C_{Z}(\alpha)+C_{Z_{q}}(\alpha) \frac{c q}{2 V_{a}}+C_{Z_{\delta e}}(\alpha) \delta_{e}\right] \\
& \dot{\phi}=p+q \sin \phi \tan \theta+r \cos \phi \tan \theta \\
& \dot{\theta}=q \cos \phi-r \sin \phi \\
& \dot{\psi}=q \sin \phi \sec \theta+r \cos \phi \sec \theta \\
& \dot{p}=\tilde{\mathrm{A}}_{1} p q-\tilde{\mathrm{A}}_{2} q r+\frac{\rho V_{a}^{2} S b}{2}\left[C_{p_{0}}+C_{p_{\beta}} \beta+C_{p_{p}} \frac{b p}{2 V_{a}}+C_{p_{r}} \frac{b r}{2 V_{a}}+C_{p_{\delta a}} \delta_{a}+C_{Y_{\delta r}} \delta_{r}\right] \\
& \dot{q}=\tilde{\mathrm{A}}_{5} p r-\tilde{\mathrm{A}}_{6}\left(p^{2}-r^{2}\right)+\frac{\rho V_{a}^{2} S c}{2 J_{y}}\left[C_{m_{0}}+C_{m_{\alpha}}+C_{m_{q}} \frac{c q}{2 V_{a}}+C_{m_{\dot{\delta} e}} \delta_{e}\right] \\
& \dot{r}=\tilde{\mathrm{A}}_{,} p q-\tilde{\mathrm{A}}_{1} q r+\frac{\rho V_{a}^{2} S b}{2}\left[C_{r_{0}}+C_{r_{\beta}} \beta+C_{r_{p}} \frac{b p}{2 V_{a}}+C_{r_{r}} \frac{b r}{2 V_{a}}+C_{r_{\delta_{\alpha}}} \delta_{a}+C_{r_{\delta_{r}}} \delta_{r}\right]
\end{aligned}
$$

Notes:

- The lift and drag terms is nonlinear in $(\alpha)$,

- The propeller thrust is nonlinear in the throttle command.

As we interested in modeling UAV flight under low angle of attack conditions, so simpler linear model can be utilized for

$$
\begin{aligned}
& C_{L}(\alpha)=C_{L 0}+C_{L \alpha} \alpha \\
& C_{D}(\alpha)=C_{D 0}+C_{D \alpha} \alpha
\end{aligned}
$$

\section{Linearization of Equations of Motion}

Model linearization is based on the small disturbance theory. According to this theory, analysis is done under small perturbations of motion characteristics [15].

Linearization and decoupling of the 12-state equations of motion to produce a reduced linear transfer functions or state space models describing the nonlinear UAV airframe is the most appreciable target to this paper. Low level autopilot control loops for UAV will be designed using This LTI system. The dynamics of aircraft is decoupled into longitudinal $(\theta, \mathrm{q}, \mathrm{h}, \mathrm{u}, \mathrm{w})$ and lateral dynamics $(\phi, \mathrm{p}, \psi, \mathrm{r}, \mathrm{v})$. The trimming algorithm at a steady state flight condition will be discussed at the following subsection.

\section{Equilibrium point and steady state flight}

Bringing the model under control is done by finding a combination of values of the state and control variables that correspond to a steadystate flight condition then decoupling of the dynamics [2], so the next step is to analyze the dynamics of the aircraft about steady state scenarios or equilibrium points which is actually called trimming technique.

The linearization condition which make $\dot{X}=0, U=0$ or constant is supposed. With these conditions the system is called to be at rest (all derivative is equal zero), then examine the behavior of the system near the equilibrium point by slightly perturbing some of the variables. Steady state aircraft flight can be defined as a condition in which all of the motion variables are constant or zero.

- Linear and angular velocities are constants or zero.

- $\quad$ All acceleration components are zero.

- $\quad$ Flat earth.

- Mass of the aircraft is constant. altitude.

Neglecting of the change of atmosphere density due to

These definitions are available for some aircraft basic scenarios [2].

- $\quad$ Steady wings level flight.

- $\quad$ Steady turning flight.

- $\quad$ Steady wings level climb.

- Climbing turn.

For steady state flight:

$$
\dot{p}, \dot{q}, \dot{r}, \dot{u}, \dot{v}, \dot{w}\left(\text { or } \dot{V}_{a}, \dot{\beta}, \dot{\alpha}\right)=0
$$

$\mathrm{U}=$ constant

With the following additional constraints according to the flight condition:

Steady wings level flight: $\phi, \dot{\phi}, \dot{\theta}, \dot{\psi}=0$

Steady turning flight: $\quad \dot{\phi}, \dot{\theta}=0$, and $\dot{\psi}$ turn rate

Steady pull-up flight : $\quad \phi, \dot{\phi}, \dot{\psi}=0$, and $\dot{\theta}$ pull-up rate

Steady climbing turn: $\quad \dot{\phi}=0, \dot{\theta}$ pull-up rate, and $\dot{\psi}$ turn rate

For a fixed wing UAV

$\begin{array}{ll}\text { The states are: } & X=\left(P_{n}, P_{e}, h, u, v, w, \phi, \theta, \psi, p, q, r\right)^{T} \\ \text { The inputs: } & U=\left(\delta_{e}, \delta_{t}, \delta_{a}, \delta_{r}\right)^{T}\end{array}$

Our knowledge of the aircraft behavior allows us to specify the required steady state condition so that the trim algorithm converges on an appropriate solution. The generic trim program links to any nonlinear model produces a file containing the steady state values for the states and control inputs for use in the linearization programs.

The aircraft designers must know how to specify the steady state condition; how many of the state and control variables may be chosen independently, and what constraints exist on the remaining variables.

In the process of performing trim calculations for the SUAV the wind will be treated as an unknown disturbance, so the wind speed is zero. The trim calculation algorithm output the trim states and inputs according to the steady state condition. These assumed steady state flights for the Ultrastick-25e as follows.

I. Steady Straight and Level flight.

II. Level Climb.

III. Level Turn.

IV. Climbing Turn.

So the trimmed outputs and controls are summarized in Table 2 
Citation: Ahmed EA, Hafez A, Ouda AN, Ahmed HEH, Abd-Elkader HM (2015) Modelling of a Small Unmanned Aerial Vehicle. Adv Robot Autom 4: 126. doi: 10.4172/2168-9695.1000126

Page 6 of 11

\begin{tabular}{|c|c|c|c|c|}
\hline & I & II & III & IV \\
\hline$\delta_{t}$ & 0.569 & 0.721 & 0.582 & 0.731 \\
\hline$\delta_{e}$ & -0.0963 & -0.102 & -0.125 & -0.131 \\
\hline$\delta_{r}$ & 0.00317 & 0.00436 & -0.00748 & -0.00607 \\
\hline$\delta_{a}$ & 0.01 & 0.0138 & 0.0186 & 0.0253 \\
\hline$V_{a}$ & 17 & 17 & 17 & 17 \\
\hline$\beta$ & $3.72 * 10^{-22}$ & $3.56^{*} 10^{-25}$ & $-1.51^{*} 10^{-20}$ & $5.8^{*} 10^{-20}$ \\
\hline$\alpha$ & 0.054 & 0.0529 & 0.0646 & 0.0633 \\
\hline$h$ & 100 & Don't care & & \\
\hline$\varphi$ & -0.00172 & -0.00239 & 0.544 & 0.547 \\
\hline$\theta$ & 0.054 & 0.14 & 0.0553 & 0.141 \\
\hline$\psi$ & 2.71 & 2.71 & 2.71 & 2.71 \\
\hline$p$ & $5.09^{*} 10^{-27}$ & $5.21^{*} 10^{-28}$ & -0.0193 & -0.0492 \\
\hline$q$ & $-7.56^{*} 10^{-23}$ & $1.12^{*} 10^{-26}$ & 0.181 & 0.18 \\
\hline$r$ & $-1.03^{*} 10^{-24}$ & $-8.32^{*} 10^{-28}$ & 0.298 & 0.295 \\
\hline$\gamma$ & $-9.8^{*} 10^{-17}$ & 0.0873 & $-1.23^{*} 10^{-09}$ & 0.0873 \\
\hline & & & & \\
\hline
\end{tabular}

Table 2: Trimmed values for ultrastick-25e (THOR).

which represents a set of trimmed conditions for the Ultrastick-25e model. These values will be used in the next section to evaluate the linear lateral and longitudinal state space models, and then used in the autopilot design according to the desired flight conditions.

\section{Linear state space model}

After obtaining nonlinear 12-state equations of motion and obtaining the trimmed values of different flight conditions, a linearization technique to linearize the equations will be derived to obtain the state space models for longitudinal and lateral dynamics at last.

Calculating the jacobian matrices for LTI equations directly from the nonlinear model are done by assigning the state and control variables from the steady state conditions, and numerically evaluating the partial derivatives in the jacobian matrices. The jacobian matrices may therefore be determined for any steady state flight condition [13]. The linearization program is done to determine A, B, C, D matrices of the state space model.

At last of this subsection the full description of a longitudinal linear state space model with its reduced order modes including the shortperiod mode, the phugoid mode. Then the description lateral model with its roll mode, the dutch-roll mode, and the spiral-divergence mode.

\section{Longitudinal state space model}

The longitudinal state equations are given by

$$
\dot{x}_{l o n} \triangleq(u, w, q, \theta, h)^{T} \text {. }
$$

And the input (control) vector is defined as:

$$
U_{\text {lon }} \triangleq\left(\delta_{e}, \delta_{t}\right)^{T} \text {. }
$$

Expressing (8), (10), (15), (12), and (7) in terms of $x_{\text {lon }}$ and $U_{\text {lon }}$, Assuming that the lateral states are zero (i.e., $\varphi=p=r=\beta=v=0$ ) and the wind speed is zero.

\section{Valued longitudinal model for straight and level flight}

State space longitudinal model has 5 States $(\mathrm{u}, \mathrm{w}, \theta$, $\mathrm{q}$, and $-\mathrm{h}), 2$ Inputs $\delta_{e}$, and $\delta_{t}$, and 5 Outputs $\left(\mathrm{V}_{\mathrm{a}}, \alpha, \theta, \mathrm{q}\right.$, and $\left.\mathrm{h}\right)$. The longitudinal linear state space model is SYS $_{\text {lon }}$ which has $\left(A_{l o n}, B_{l o n}, C_{l o n}, D_{l o n}\right)$.

$$
-\boldsymbol{A}_{\text {lon }}=\left(\begin{array}{llrrcc}
-0.5944 & 0.8008 & -9.791 & -0.8747 & 5.077 * 10^{-5} \\
-0.744 & -7.56 & -0.5294 & 15.72 & -0.000939 \\
0 & 0 & 0 & 1 & 0 \\
1.041 & -7.406 & 0 & -15.81 & -7.284 * 10^{-18} \\
-0.05399 & 0.9985 & -17 & 0 & 0
\end{array}\right)
$$$$
-\boldsymbol{B}_{\text {lon }}=\left(\begin{array}{ll}
0.4669 & 0 \\
-2.703 & 0 \\
0 & 0 \\
-133.7 & 0 \\
0 & 0
\end{array}\right)
$$

$$
-C_{\text {lon }}=\left(\begin{array}{lllll}
0.9985 & 0.05399 & 0 & 0 & 0 \\
-003176 & 0.05874 & 0 & 0 & 0 \\
0 & 0 & 0 & 1 & 0 \\
0 & 0 & 1 & 0 & 0 \\
0 & 0 & 0 & 0 & -1
\end{array}\right)
$$

$$
\text { - } \quad D_{\text {lon }}=0
$$

The eigenvalues can be determined by finding the eigenvalues of the matrix $A_{\text {lon }}$

$$
|\lambda I-A|=0
$$

Longitudinal Poles:

$\begin{array}{lcl}\text { Eigenvalue } & \text { Damping } & \text { Frequency } \\ -0.159 \pm 0.641 \mathrm{i} & 0.241 & 0.66 \text { (phugoid) } \\ -11.7 \pm 10.0 \mathrm{i} & 0.759 & 15.4 \text { (short period) }\end{array}$

The reduced order modes of the longitudinal dynamics are the approximation of the full linear longitudinal model. At phugoid mode or long period mode is a lightly damped and slow response to the inputs but the short period mode is fast and acceptable [15]

- The linearized outputs (v,a, q, $\theta, \mathrm{h})$ response due to $\bar{\delta}_{e}$ input are represented in polynomial form equations $17-(a, b, c, d, e)$.

$\frac{\overline{v_{a}}(S)}{\overline{\delta_{e}}(S)}=\frac{0.3203 S^{5}+11.53 S^{4}+555 S^{3}+12630 S^{2}+57190 S-15.45}{S^{6}+29.86 S^{5}+391 S^{4}+1598 S^{3}+632.6 S^{2}+638.1 S+0.07671}$

$\frac{\bar{\alpha}(S)}{\bar{\delta}_{e}(S)}=\frac{-0.1603 S^{5}-127.4 S^{4}-823.8 S^{3}-330.8 S^{2}-521.1 S-0.07907}{S^{6}+29.86 S^{5}+391 S^{4}+1598 S^{3}+632.6 S^{2}+638.1 S+0.07671}$

$\frac{\bar{q}(S)}{\bar{\delta}_{e}(S)}=\frac{-133.7 S^{5}-1859 S^{4}-6751 S^{3}-2245 S^{2}-0.07907 S-2.806^{*} 10^{-16}}{S^{6}+29.86 S^{5}+391 S^{4}+1598 S^{3}+632.6 S^{2}+638.1 S+0.07671}$

$\frac{\bar{\theta}(S)}{\bar{\delta}_{e}(S)}=\frac{-133.7 S^{4}-1859 S^{3}-6751 S^{2}-2245 S-0.07907}{S^{6}+29.86 S^{5}+391 S^{4}+1598 S^{3}+632.6 S^{2}+638.1 S+0.07671}$

$\frac{\bar{h}(S)}{\bar{\delta}_{e}(S)}=\frac{2.725 S^{4}-106.8 S^{3}-17590 S^{2}-109100 S-29300}{S^{6}+29.86 S^{5}+391 S^{4}+1598 S^{3}+632.6 S^{2}+638.1 S+0.07671}$ 


\section{Lateral state space model}

Lateral directional equations of motion consist of the side force, rolling moment and yawing moment equations of motion. For the lateral state-space equations, the state is given by

$$
\dot{x}_{\text {lat }} \triangleq(v, p, r, \phi, \psi)^{T}
$$

And the input (control) vector is defined as:

$$
U_{\text {lat }} \triangleq\left(\delta_{a}, \delta_{r}\right)^{T}
$$

Expressing equations (9), (14), (16), (11), and (13) in terms of $x_{\text {lat }}$ and $U_{\text {lat }}$, we get The Jacobians of equations.

\section{Valued lateral model for straight and level flight}

The lateral-directional model has five states (v, p, r, $\phi, \psi)$, two inputs $\left(\delta_{a}, \delta_{r}\right)$, and five outputs $(\beta, \mathrm{p}, \mathrm{r}, \phi, \psi)$. The lateral state space model is $\mathrm{SYS}_{\text {lat }}$ with $\left(A_{\text {lat }} B_{\text {lat }}, C_{\text {lat }}, D_{\text {lat }}\right)$.

$$
\begin{aligned}
A_{\text {lat }} & =\left(\begin{array}{llccc}
-0.8726 & 0.8789 & -16.82 & 9.791 & 0 \\
-2.823 & -16.09 & 3.367 & 0 & 0 \\
0.702 & 0.514 & -2.775 & 0 & 0 \\
0 & 1 & 0.05406 & -4.088^{*} \cdot 10^{-24} & 0 \\
0 & 0 & 1.001 & -7.573 * 10^{-23} & 0
\end{array}\right) \\
\boldsymbol{B}_{\text {lat }} & =\left(\begin{array}{ll}
0 & 5.302 \\
-156.5 & -5.008 \\
11.5 & -82.04 \\
0 & 0 \\
0 & 0
\end{array}\right) \\
\boldsymbol{C}_{\text {lat }} & =\left(\begin{array}{ll}
0.05882 & 0000 \\
0 & 1000 \\
0 & 0100 \\
0 & 0010 \\
0 & 0001
\end{array}\right) \\
\boldsymbol{D}_{\text {lat }} 0 &
\end{aligned}
$$

The null column in the $A_{\text {lat }}$ matrix shows that the state $\psi$ is not coupled back to any other states, and it can be omitted from the state equations when designing the stability augmentation system.

The eigenvalues can be determined by finding the eigenvalues of the matrix A.

$$
|\lambda I-A|=0
$$

Lateral-Directional Poles:

$\begin{array}{lcc}\text { Eigenvalue } & \text { Damping } & \text { Frequency } \\ 0 & -1 & 0 \\ -.0138 & 1.00 & 0.0138 \text { (spiral) } \\ -1.84 \pm 5.28 \mathrm{i} & 0.329 & 5.59 \text { (dutch roll) } \\ -16.1 & 1.00 & 16.1 \text { (roll) }\end{array}$

In general we found that the roots of the lateral-directional characteristic equation composed of two real roots and a pair of complex roots. These roots will characterize the airplane response [16].

The dutch roll poles are not canceled out of the transfer function $p / \delta_{a}$ complex zeros, thus meaning that Coupling exists between the rolling and yawing motions, the dutch roll mode will involve some rolling motion. These transfer functions validate the decision to use the MIMO state equations for the analysis, so the linearized outputs $(\beta, p, r, \phi)$ response due to $\bar{\delta}_{a}$ input are the eqns. $\left.18-(\mathrm{a}, \mathrm{b}, \mathrm{c}, \mathrm{d})\right)$.

$$
\begin{aligned}
& \frac{\bar{\beta}(S)}{\bar{\delta}_{a}(S)}=\frac{-19.47 S^{2}-213.8 S-224.5}{S^{4}+19.74 S^{3}+90.49 S^{2}+502.2 S+6.89} \\
& \frac{\bar{p}(S)}{\bar{\delta}_{a}(S)}=\frac{-156.5 S^{3}-532 S^{2}-4277 S+123.7}{S^{4}+19.74 S^{3}+90.49 S^{2}+502.2 S+6.89} \\
& \frac{\bar{r}(S)}{\bar{\delta}_{a}(S)}=\frac{11.5 S^{3}+114.8 S^{2}-114.1 S-2289}{S^{4}+19.74 S^{3}+90.49 S^{2}+502.2 S+6.89} \\
& \frac{\bar{\phi}(S)}{\overline{\delta_{a}}(S)}=\frac{-155.8 S^{2}-525.8 S-4283}{S^{4}+19.74 S^{3}+90.49 S^{2}+502.2 S+6.89}
\end{aligned}
$$

And the linearized outputs $(\beta, p, r, \phi)$ response due to $\bar{\delta}_{r}$ input are the eqns. 19-(a, b, c, d)).

$$
\begin{gathered}
\frac{\bar{\beta}(S)}{\overline{\delta_{r}}(S)}=\frac{0.3119 S^{3}+86.8 S^{2}+1302 S-208.3}{S^{4}+19.74 S^{3}+90.49 S^{2}+502.2 S+6.89} \\
\frac{\bar{p}(S)}{\overline{\delta_{r}}(S)}=\frac{-5.008 S^{3}-309.5 S^{2}-4304 S+127.1}{S^{4}+19.74 S^{3}+90.49 S^{2}+502.2 S+6.89} \\
\frac{\bar{r}(S)}{\bar{\delta}_{r}(S)}=\frac{-82.04 S^{3}-1385 S^{2}-1228 S-2351}{S^{4}+19.74 S^{3}+90.49 S^{2}+502.2 S+6.89} \\
\frac{\bar{\phi}(S)}{\overline{\delta_{r}}(S)}=\frac{-9.443 S^{2}-384.4 S-4370}{S^{4}+19.74 S^{3}+90.49 S^{2}+502.2 S+6.89}
\end{gathered}
$$

\section{Analytical linearization of aircraft equations of motion}

In this section the analytical linearization of roll and roll dynamics can be derived to check the matching between state space linearized model and the analytical model.

\section{First: roll or bank angle $(\phi)$}

The equation (11) can be considered to be linearized from this main assumption which is logic for most flight conditions; the pitch angle $(\theta)$ is a small this means that the primarily influence on $\phi$ equation is roll rate $(p)[8]$, so

$$
\dot{\phi}=p+d_{\phi 1}
$$

Second: differentiate the above equation we get:

$\phi=\dot{p}+\dot{d}_{\phi_{1}}$

Third: substitute $p$ by eqn. (14) and the equation in the first step we 
Citation: Ahmed EA, Hafez A, Ouda AN, Ahmed HEH, Abd-Elkader HM (2015) Modelling of a Small Unmanned Aerial Vehicle. Adv Robot Autom 4: 126. doi: 10.4172/2168-9695.1000126

Page 8 of 11

will get the equation as follows

$$
\phi=-a_{\phi 1} \dot{\phi}+a_{\phi 2} \delta_{a}+d_{\phi_{2}}
$$

Where:

$a_{\phi 1}, a_{\phi 2}$ are the coefficients of the roll dynamics, they are variables in the aircraft parameters and the trimmed values.

$d_{\phi_{2}}$ can be considered as a disturbance on the system.

Fourth: Laplace transfer function is as follows:

$$
\phi(s)=\frac{a_{\phi 2}}{s\left(s+a_{\phi 1}\right)}\left(\delta_{a}(s)+\frac{1}{a_{\phi 2}} d_{\phi 2}(s)\right)
$$

Fifth: the final numerical transfer function of roll $(\phi)$ for $\delta_{a}$ as input is as follows:

$$
\phi(s) \approx \frac{-163.6}{s(s+16.82)} \delta_{a}(s)
$$
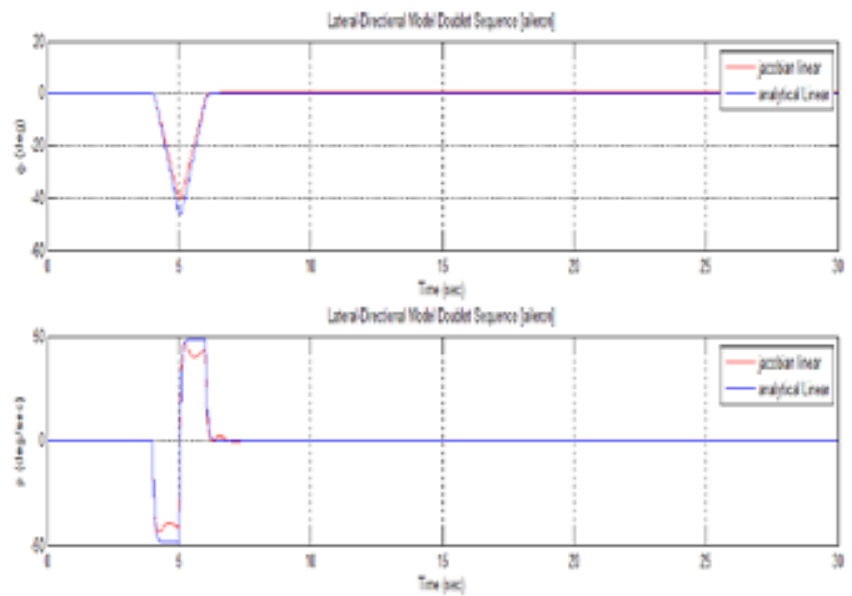

Figure 7: Linearized roll and roll rate comparison techniques with applying the doublet signal at the control surface $\left(\delta_{\mathrm{a}}\right)$.
Sixth: roll rate $(p)$ can be approximately considered as the differentiation of the roll angle so

$$
p(s) \approx \frac{-163.6}{(s+16.82)} \delta_{a}(s)
$$

Comparison between the analytical and state space linearization by jacobian matrices is as follows:

Figure 7 illustrates the good matching between the two linearization techniques. The next section illustrates how to validate the linear model.

\section{Validation of Aircraft Model Linearization}

After getting the model some checks of the Ultrastick-25e (thor) longitudinal dynamics responses to (elevator) and lateral dynamics responses to (aileron, rudder) deflections of linear and nonlinear models will be illustrated in the following figures by applying a doublet pulse (a pulse that is symmetric about its reference level (the trim setting) to the control inputs) to see the response of the various outputs.

\section{Doublet response of the linear and nonlinear longitudinal model}

Doublet response of the longitudinal dynamics $\left(v_{a}, \alpha, q, \theta, h\right)$ of the linear model and nonlinear model is shown in the following Figures 8-10.

\section{Doublet response of the linear and nonlinear lateral model}

Doublet response of the lateral dynamics outputs $(\beta, p, r, \phi, \psi)$ response due to doublet $\bar{\delta}_{a}, \bar{\delta}_{r}$ of the linear model and nonlinear model (simulink) are shown in the following Figures 11 and 12.

\section{Conclusion}

The objective of this paper is achieved by development of an accurate mathematical model for SUAV. The resulted mathematical model is an efficient and very compatible for designing an accurate autopilot with various phases of flights. The nonlinear equations of motion extraction are focused. With these equations the linear longitudinal and lateral models are obtained. The methodology of getting the linear model is validated by comparing between two linearization methods then

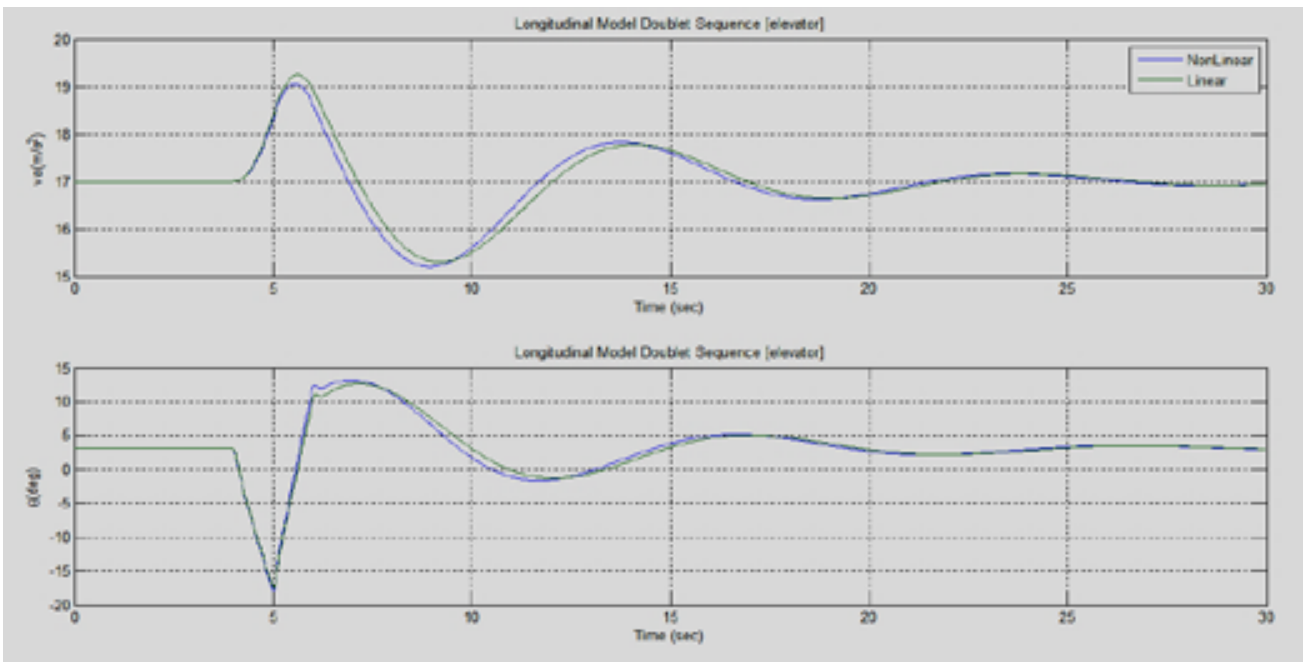

Figure 8: Response of $(V \alpha, \theta)$ of Ultrastick-25e model due to elevator doublet (trim \pm 5 degree). 

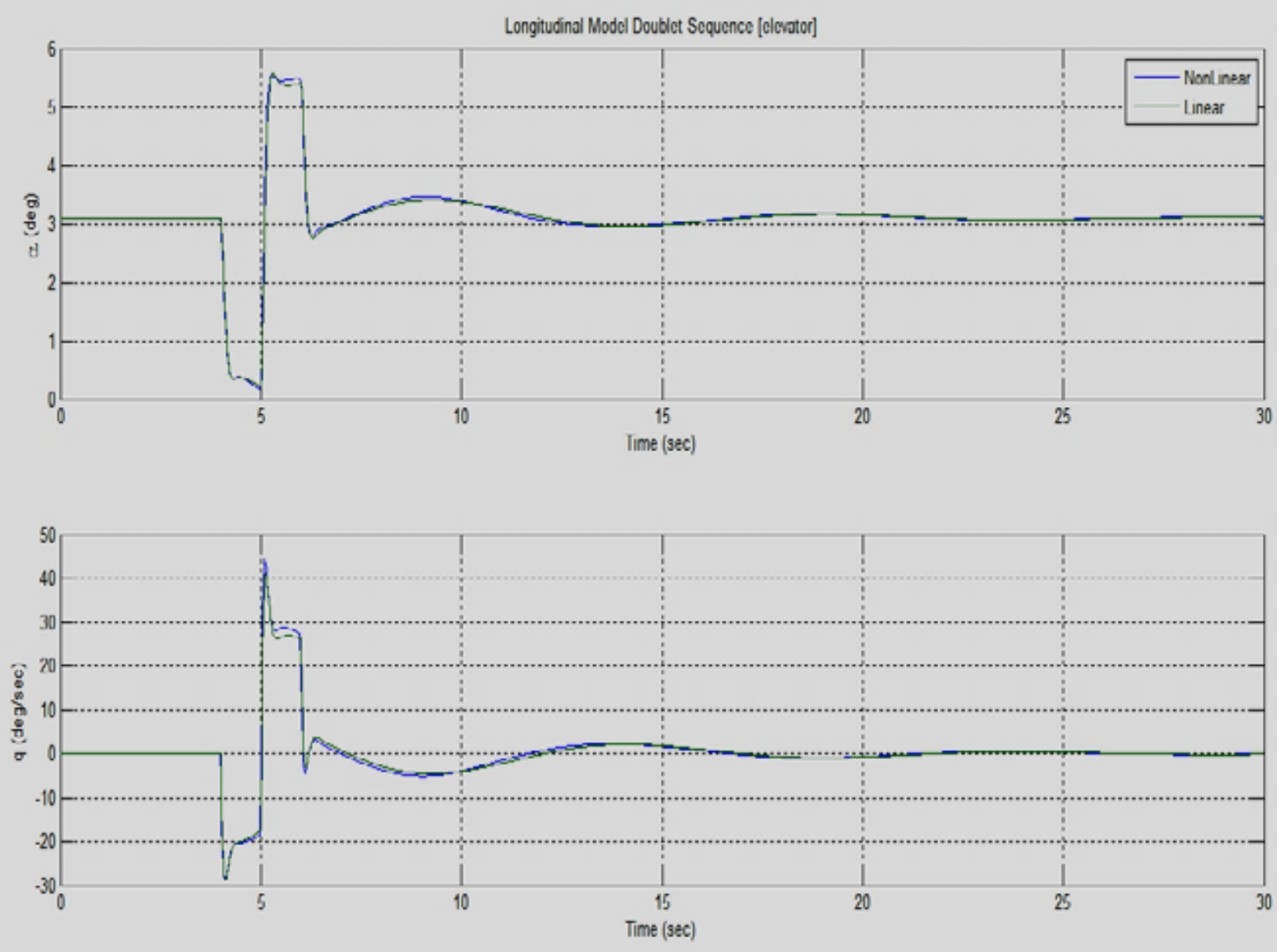

Figure 9: Response of $(a, q)$ of Ultrastick-25e model due to elevator doublet (trim \pm 5 degree).

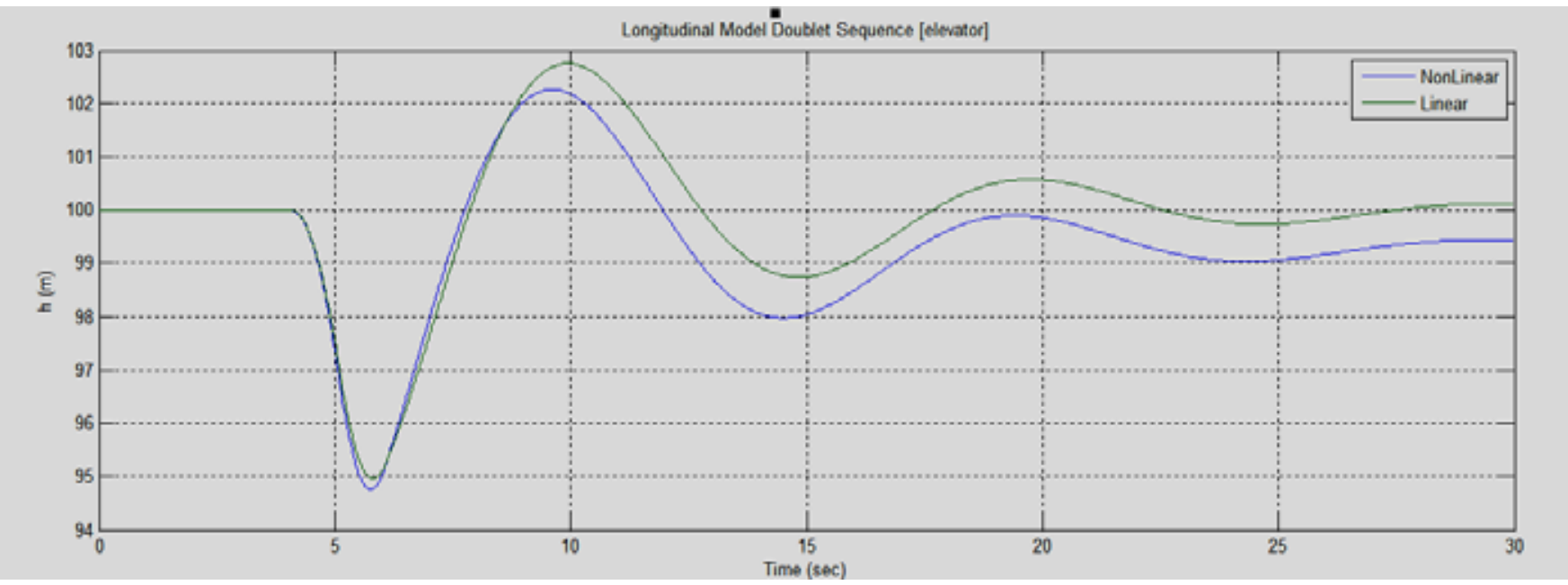

Figure 10:Response of $(h)$ of Ultrastick-25e model due to elevator doublet (trim \pm 5 degree). 
Citation: Ahmed EA, Hafez A, Ouda AN, Ahmed HEH, Abd-Elkader HM (2015) Modelling of a Small Unmanned Aerial Vehicle. Adv Robot Autom 4: 126. doi: 10.4172/2168-9695.1000126
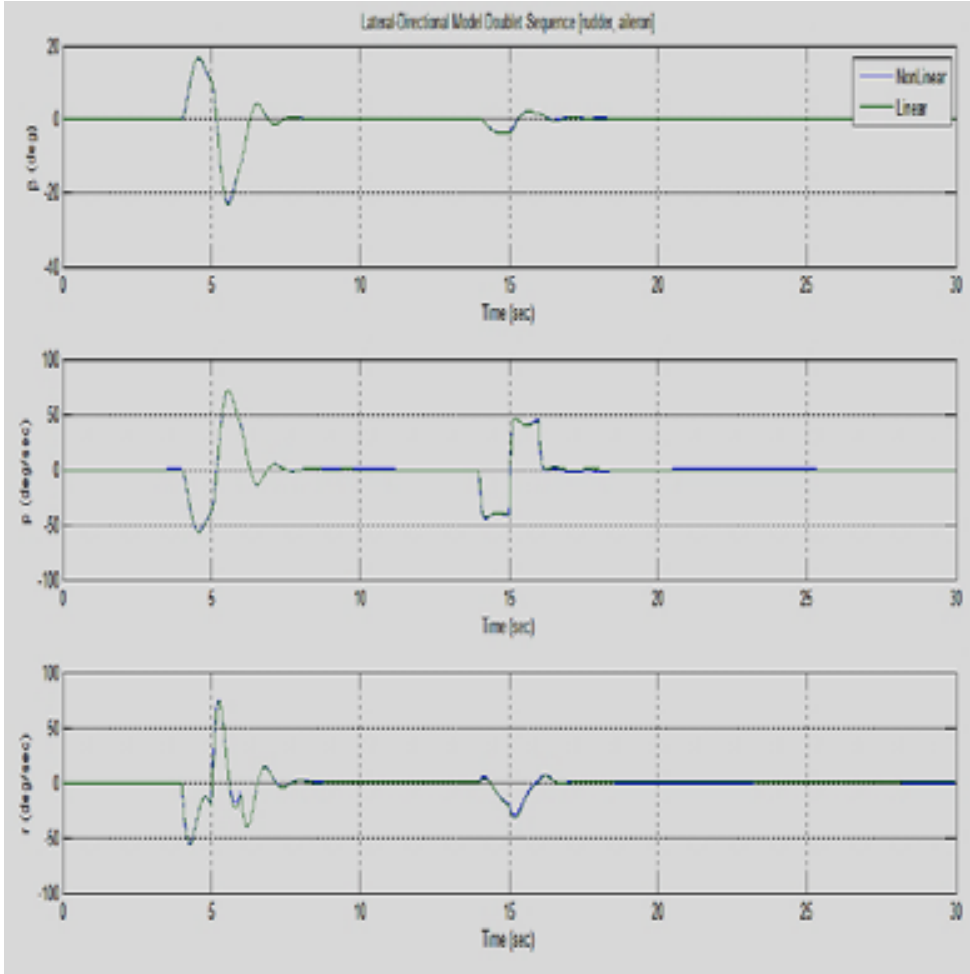

Figure 11: Response of the lateral dynamics $(\beta, p, r)$ due to $5^{\circ}$ (aileron, rudder) deflection doublet signal.

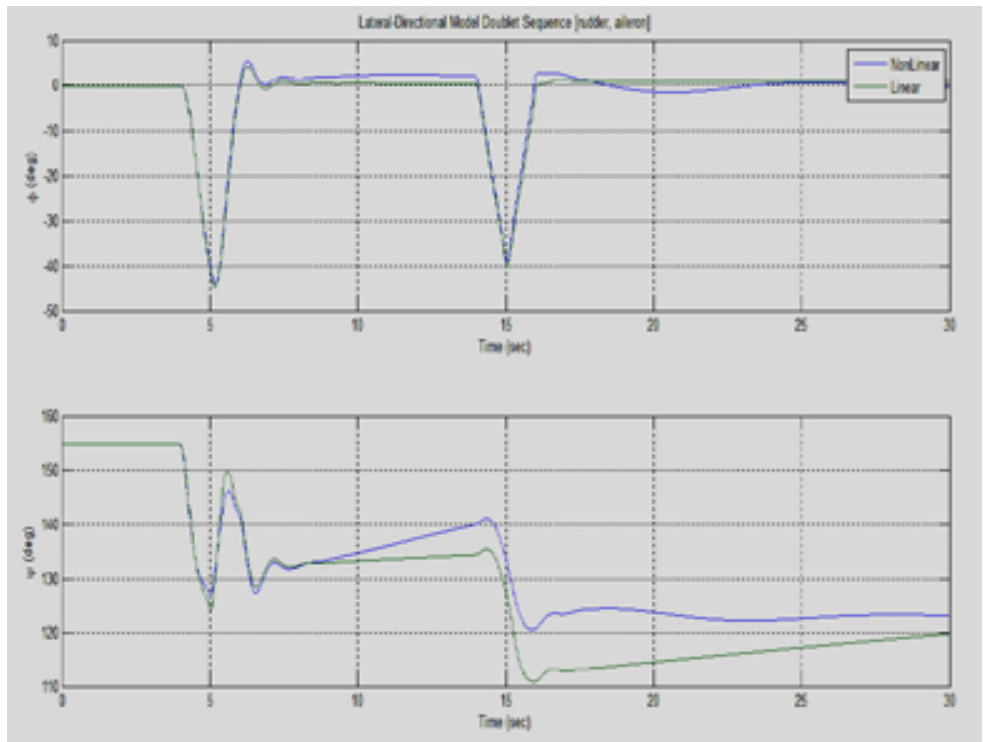

Figure 12: Response of the lateral dynamics $(\phi, \psi)$ due to $5^{\circ}$ (aileron, rudder) deflection doublet signal.

comparing the most accurate one with the nonlinear state equations. The linearized model can approximately describe the behavior of the nonlinear dynamics of SUAV. At the future work when designing the autopilot we will use this linearized lateral and longitudinal models to design the controller gains of inner and outer loops of autopilot.

\section{References}

1. Edward AFB CA (1988) Flying Qualities Phase. USAF TEST Pilot School.
2. Stevens BL, Lewis FL (2003) Aircraft Control and Simulation. (2 nd ed) JohnWiley and Sons.

3. Greenwood DT (1988) Principles of Dynamics. (2nd ed ) NJ: Prentice Hall.

4. Williams JE (1980) USAF Stability and Control DATCOM. Air Force Flight Dynamics Laboratory, Wright Patterson Air Force Base.

5. Xinzhong C, Ahsan K (2002) Advances in Modeling of Aerodynamic Forces on Bridge Decks. Journal of Engineering Mechanics 1193-1205.

6. Murch A, Dorobantu A, Balas G (2013) University of Minnesota UAV Flight Control Research Group. 
Citation: Ahmed EA, Hafez A, Ouda AN, Ahmed HEH, Abd-Elkader HM (2015) Modelling of a Small Unmanned Aerial Vehicle. Adv Robot Autom 4: 126. doi: 10.4172/2168-9695.1000126

7. Murch A, Paw YC, Pandita R, Li Z, Balas G (2011) A Low Cost Small UAV Flight Research Facility. CEAS Conference on Guidance, Navigation, and Control, Munich, Germany.

8. Beard RW, Mclain TW (2012) Small Unmanned Aircraft: Theory and Practice. Princeton university press.

9. Beal TR (1993) Digital Simulation Of Atmospheric Turbulence For Dryden And Von Karman Models. Journal of Guidance, Control, and Dynamics 16: 132-138.

10. Nelson RC (1998) Flight Stability and Automatic Control. (2nd ed) MA:McGrawHill, Boston.

11. Coughey DA (2011) Introduction To Aircraft Stability And Control. Sibley Schoo of Mechanical and Aerospace Engineering, Cornell University, Ithaca, New York.
12. Noth A, Bouabdallah S, Siegwart R (2006) Dynamic Modeling of Fixed-Wing UAVs. Swiss Federal institute of technology, Maxim Antipov.

13. Jodeh NM (2006) Development of Autonomous Unmanned Aerial Vehicle Research Platform: Modeling, Simulating, And Flight Testing, Air Force Institute of Technology, Wright-Patterson Air Force Base,

14. Cook MV (2012) Flight Dynamics Principles: A Linear Systems Approach to Aircraft Stability and Control. ( $3^{\text {rd }}$ Ed) Elsevier Ltd.

15. Valavanis KP (2007) Advances In Unmanned Aerial Vehicles. University of South Florida, Tampa, Florida, USA

16. Hull DG (2005) Fundamentals of Airplane Flight Mechanics. Austin, Texas. 